\title{
POLÍTICAS PÚBLICAS
}

\section{Segmentación y fragmentación del Sistema Nacional de Salud desde la percepción de actores políticos-sociales. San Lorenzo, Paraguay, 2017}

\author{
Celso Obdulio Mora Rojas ${ }^{1}$
}

\section{Resumen}

Introducción: La salud es un estado de bienestar completo y un sistema de salud engloba a todas las organizaciones, instituciones y recursos cuyo principal objetivo es llevar a cabo actividades encaminadas a mejorar la salud. La segmentación del sistema de salud es entendida como la presencia de diversos subsistemas responsables de atención en salud de diferentes grupos poblacionales y ausencia de coordinación entre los sistemas institucionales públicos, privados y de seguridad social, los sistemas de salud deben responder a necesidades de la población y el fortalecimiento de dichos sistemas son fundamentales para el desarrollo y lucha contra la pobreza. La fragmentación es considerada falta de integración y coordinación entre niveles de atención en salud. Los sistemas de salud en América Latina, continúan siendo inequitativos. Objetivo: Determinar percepción de actores políticos-sociales sobre la segmentación y fragmentación del Sistema Nacional de Salud en Paraguay.

Material y Método: Diseño fue observacional de corte transversal con enfoque cualitativo. La población estuvo constituida por personas que desempeñaron funciones de toma de decisiones en el Ministerio de Salud Pública o en otros sectores del Gobierno relacionados con la problemática, constituida por 20 personas consideradas actores políticos-sociales. Se realizaron entrevistas a profundidad, con guion estructurado de preguntas, registrando el discurso de los entrevistados; con autorización de los mismos. La participación de los sujetos de estudios fue anónima, voluntaria y confidencial. Respetando los principios de la investigación.

Resultados: El Sistema Nacional de Salud del Paraguay creado por Ley, con una concepción oportuna; la problemática es la implementación y se caracteriza por la alta segmentación y fragmentación. En Paraguay se tienen varios subsistemas con diferentes regímenes de financiación y de afiliación, con escasa o casi nula articulación entre las instituciones que ofertan servicios en el sistema, llegando

1. Universidad Nacional de Asunción. Facultad de Ciencias Químicas.

Tesis presentada al Instituto Dr. Andrés Barbero, UNA, Maestría en Gerencia y Administración de Servicios y Sistemas de Salud. San Lorenzo, Paraguay. Agosto, 2017.

E-mail: cmoraroj@gmail.com

DOI: $10.26885 /$ rcei.foro.2018.112 


\section{Segmentación y fragmentación del Sistema Nacional de Salud. Mora Rojas}

hasta la consideración de que en el país se cuenta con un No Sistema o la constancia del Sistema en documentos.

Se ha percibido que existe desconcentración y no descentralización; a pesar de la base constitucional de la descentralización, así como la coincidencia de falta de equidad en los diferentes grupos poblacionales en accesos a los sistemas de servicios en salud. Algunas barreras fueron abolidas como la arancelaria, otras persisten como las geográficas, culturales y educativas.

Otra percepción hallada fue que el concepto de equidad en cuanto a oposición de gratuidad y por otro lado a la igualdad de respuestas.

Conclusiones: La percepción de actores sociales y políticos es el sistema de salud ampliamente fragmentado y segmentado.

La descentralización tiene apoyo constitucional, debe ir avanzando.

El acceso a la salud presenta inequidad, pues no da respuestas iguales a problemas iguales. Las barreras culturales y educativas al acceso a la salud son numerosas, el denominador común es la falta de educación formal.

La fragmentación y segmentación podrían solucionarse con un amplio debate nacional, fuerte voluntad política, aplicación de redes integradas de salud, según el modelo de sistema de salud que la comunidad paraguaya opte.

Palabras clave: sistema nacional de salud, fragmentación, segmentación.

\section{Referencias}

Flecha, O., Ortellado, J., Gaete, R., Martínez, E., \& Carrizosa, A. (1996). Diagnóstico sobre la Situación del Sector Salud en el Paraguay. Serie Estudios 11-CEPRO, 54-80.

Organización Mundial de la Salud. (9 de Noviembre de 2005). ¿Qué es un Sistema de Salud? Recuperado de http://www.who.int/features/qa/28/es/

Organización Panamericana de la Salud. (28 de Mayo de 2010). Organización Panamericana de la Salud-Redes Integradas de Servicios de Salud: Conceptos, Opciones de Política y Hoja de Ruta para su Implementación en las Américas. Recuperado de https://www.paho.org/uru/ index.php?option=com_docman\&view=download\&alias $=145$ redes-integradas-de-servicios-de-salud-aps-n4\&category_ slug=publicaciones-sistemas-y-servicios-de-salud\&ltemid=307

Organización Paramericana de la Salud/USAID. (s/d de Enero de 2008). Perfi I de los Sistemas de Salud de Paraguay: Monitoreo y análisis de los procesos de cambio y reforma. Recuperado de www1.paho.org/hq/ dmdocuments/2010/Perfil_Sistema_Salud-Paraguay_2008.pdf

Scialabba, E. (8 de Mayo de 2013). Sistemas de salud en América Latina: financiamiento y gasto. Recuperado de http://elianascialabba. wordpress.com/2015/05/08sistemas-de-salud-en américa- latinafinanciamiento-y gasto. 